\title{
Monitoring drug crystallization in percutaneous penetration using localized nano-thermal analysis and photothermal microspectroscopy
}

Choon Fu Goh ${ }^{\text {a, b, }}{ }^{*}$, Jonathan G Moffat ${ }^{c}$, Duncan Q M Craig ${ }^{a}$, Jonathan Hadgraft ${ }^{\text {a }}$, Majella E Lane ${ }^{a}$

a Department of Pharmaceutics, UCL School of Pharmacy, 29-39 Brunswick Square, London WC1N 1AX United Kingdom

b School of Pharmaceutical Sciences, Universiti Sains Malaysia, Penang 11800, Malaysia

c Asylum Research, an Oxford Instruments Company, Halifax Road, HP12 3SE, High Wycombe, United Kingdom

\section{* Corresponding author:}

Choon Fu Goh

School of Pharmaceutical Sciences, Universiti Sains Malaysia, Penang 11800, Malaysia Phone: +604-6532074. Fax: +604-6570017. Email: gohchoonfu@hotmail.com /

choonfugoh@usm.my 


\section{KEYWORDS}

Stratum corneum; crystallization; tape stripping; nano-thermal analysis; photothermal microspectroscopy

\section{GRAPHICAL ABSTRACT}

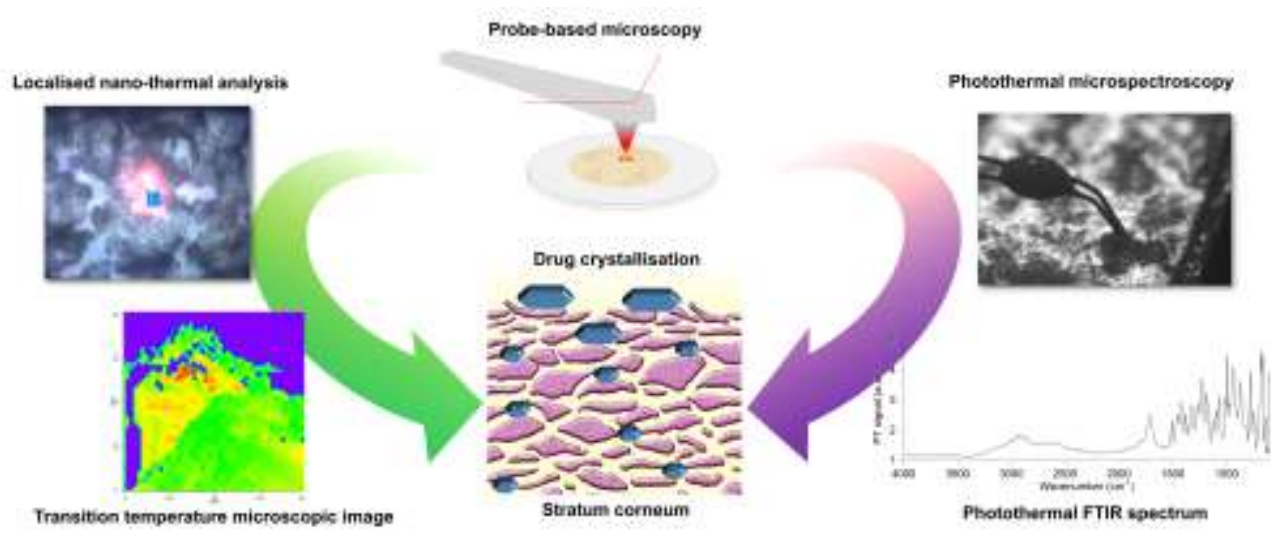

\section{ABSTRACT}

Drug crystallization on and in the skin has been reported following application of topical or transdermal formulations. This study explored novel probe-based approaches including localized nano-thermal analysis (nano-TA) and photothermal microspectroscopy (PTMS) to investigate and locate drug crystals in the stratum corneum (SC) of porcine skin following application of simple ibuprofen (IBU) formulations. We also conducted in vitro skin permeation studies and tape stripping. The detection of drug crystals in the SC on tape strips was confirmed using localized nano-TA, based on the melting temperature of IBU. The melting of IBU was also evident as indicated by a double transition, and confirmed the presence of drug crystals in the SC. The single point scans of PTMS on the tape strips allowed collection of the photothermal FTIR spectra of IBU, confirming the existence of drug crystals in the skin. The combined methods also indicated that drug crystallized in the SC at a depth of $\sim 4-$ 
$7 \mu \mathrm{m}$. Future studies will examine the potential of these techniques to probe crystallization of other commonly used actives in topical and transdermal formulations.

\section{INTRODUCTION}

Human skin not only is a natural protective layer against microorganisms, but also is a major barrier for drugs to overcome. From Fick's first law of diffusion an important factor in promoting molecular diffusion is the chemical potential of the drug in the formulation. Drug flux increases linearly with concentration until the solubility limit in the formulation is attained. Theoretically, a high concentration gradient should increase the thermodynamic activity of the drug. Therefore, a simple and non-invasive approach to deliver more drug into the skin is to increase drug concentration (solubility) in a formulation, even beyond its saturated solubility or supersaturation ${ }^{1}$. However, such systems are unstable and may result in crystallization of the active, ultimately limiting drug movement through the skin.

Several reports have shown drug crystallization as a possible cause of poor drug permeation in the $\operatorname{skin}^{2-6}$. In order to examine this problem, we recently used ATR-FTIR spectroscopy combined with multivariate analysis and showed the formation of drug crystals, mainly in the superficial layers of the stratum corneum, SC $(\sim 0.7-1.3 \mu \mathrm{m})^{7}$. However, the technique of ATR-FTIR spectroscopy only allows examination of the outer layers of the skin. To probe crystallization deeper in the stratum corneum the aim of the present work was to employ two probe-based microscopy techniques - transition temperature microscopy (TTM) and photothermal microspectroscopy (PTMS) .

Atomic force microscopy (AFM) has been widely used for topographical characterization and localized force mapping ${ }^{8}$. AFM works by using a fine probe tip to scan the sample surface, allowing access to electronic state and high spatial resolution data. Incorporation of thermal analysis with AFM enables the determination of the local transition temperature of a substrate. This technique working at the nano-scale is described as localized nano-thermal analysis (nano-TA) ${ }^{9}$. TTM is also a type of localized nano-TA, visualising local thermal transitions of a sample in a color coded map. 
The transition of the temperature at each point is observed via the derivative of the displacement measurement. As the temperature of the probe increases, a positive derivative is detected due to thermal expansion but soon it drops to a negative derivative when the tip penetrates the sample. The probe is then allowed to cool after retracting from the surface. In our previous report, this technique has been successfully employed to characterise thermal properties of porcine SC at the nano-scale with and without application of propylene glycol (PG) ${ }^{10}$.

Following the evolvement of thermal probes in localized thermal analysis, PTMS was developed by incorporating a conventional Fourier transform infrared (FTIR) spectrometer into the AFM instrument as a heating source for the system ${ }^{11}$. Absorption of infrared (IR) radiation by the sample produces small temperature changes which is detected by the thermal probe, primarily the Wollaston wire thermal probe ${ }^{12-14}$. The function of the Fourier transform is to enable conversion of the signal produced by the probe into a new spectrum similar in nature to typical IR spectra. The combination of FTIR spectroscopy with AFM conserves the functionality of AFM in generating images with high (sub-micron) spatial resolution. In addition, the use of FTIR spectroscopy allows recording of IR spectral data, complementing the thermal topographical profile and localized thermomechanical analysis obtained from the regions of interest in the sample.

These two probe-based microscopies have been applied extensively in pharmaceutical analysis for characterisation of various materials, differentiation of amorphous and crystalline forms ${ }^{15-20}$, examination of drug-excipient interaction and compatibility ${ }^{21,22}$ as well as investigation of topographic properties of samples with high spatial resolution of images and 3D information ${ }^{16}$.

In this paper, we applied both thermal and spectroscopic probe-based microscopies, TTM and PTMS, to investigate and monitor drug crystals formed in the SC after the permeation of ibuprofen (IBU) in simple co-solvent systems of propylene glycol (PG) and water. The formation of crystalline drug in the skin was examined as a function of depth by tape stripping the SC layers. The heterogeneity of the skin samples containing drug crystals was mapped using TTM based on the local thermal transitions of different 
materials. The presence of drug crystals on these skin samples was further confirmed with photothermal FTIR spectra measured using PTMS.

\section{MATERIALS AND METHODS}

\section{Materials}

Ibuprofen (IBU) was a gift from Wyeth Pharmaceuticals (Havant, UK). Propylene glycol (PG) was purchased from Sigma-Aldrich (Gillingham, UK). HPLC grade water, acetonitrile, methanol and trifluoroacetic acid (TFA) were obtained from Fischer Scientific (Loughborough, UK). Hydrochloric acid (1M) was provided by Alfa Aesar (Ward Hill, MA, US). Fresh porcine ears were obtained from a local abattoir and processed as described in previous work ${ }^{7}$. D-Squame ${ }^{\circledR}$ adhesive tapes (diameter of 22 $\mathrm{mm}$ and area of $3.8 \mathrm{~cm}^{2}$ ) were obtained from CuDerm Corp. (Dallas, TX, US). Porcine stratum corneum (PSC) sheets were prepared and hydrated as described previously by Tanojo, et al. ${ }^{23}$.

\section{Preparation of saturated solutions}

Saturated solutions of IBU were prepared by adding excess IBU to neat PG, water and PG:water $(75: 25,50: 50,25: 75)$ in a $2 \mathrm{~mL}$ Eppendorf tube and agitating the tubes in an oven for $48 \mathrm{~h}$ at $32 \pm 1{ }^{\circ} \mathrm{C}{ }^{24,25}$. The solutions were centrifuged to obtain supernatant for solubility determination using HPLC.

\section{In vitro permeation studies, tape stripping and IR densitometry for protein quantification using porcine ear skin}

The permeation experiments were conducted using Franz-type diffusion cells with porcine ear skin having a diffusional area of approximately $1 \mathrm{~cm}^{2}$ (accurately measured) and receptor volumes of $\sim 5 \mathrm{~mL}$. The porcine skin was allowed to thaw prior to the experiment and excess fat was removed by blunt dissection. This skin was cut into a size suitable for the Franz-type diffusion cells. Vacuum grease was applied on both 
donor and receptor chambers of diffusion cells after skin was mounted to ensure a leakproof seal between the skin and the cell components. Once assembled, receptor chambers were filled with degassed phosphate buffer saline (PBS) and a Teflon coated magnetic stirrer to ensure good mixing. The diffusion cells were then placed in a water bath for $30 \mathrm{~min}$ at $34.5 \pm 0.5^{\circ} \mathrm{C}$ on a submersible magnetic stirring bed to maintain the skin and the donor phase at $32 \pm 0.5^{\circ} \mathrm{C}$. The donor compartments were filled with 2 $\mu \mathrm{L} / \mathrm{cm}^{2}$ of the saturated solution of IBU in neat PG, water and PG:water (75:25, 50:50, 25:75). An application volume of up to $10 \mu \mathrm{L} / \mathrm{cm}^{2}$ is considered to be a finite dose ${ }^{26}$. At predetermined time intervals (every $2 \mathrm{~h}$ during the first $10 \mathrm{~h}$ with final sample collection at $24 \mathrm{~h}$ ), a volume of $0.2 \mathrm{~mL}$ of the receptor phase was removed and replaced with an equal amount of PBS. The samples were quantified using HPLC as described in section 2.2.7. Control studies with and without application of PG were carried out using the same experimental design. The number of experiment for the permeation study was $\geq 3$. In vitro tape stripping of porcine skin using D-Squame ${ }^{\circledR}$ tape (a total of 20 tape strips for each sample) and IR densitometry for SC protein quantification was carried out for all samples as reported previously ${ }^{10}$. On completion of tape stripping, the tapes were analysed using IR densitometer SquameScan ${ }^{\circledR} 850 \mathrm{~A}$ at a wavelength of $850 \mathrm{~nm}$ (Heiland Electronic GmbH, Wetzlar, Germany). The SC protein mass was calculated from the absorption values from IR densitometry using equation given, absorption (\%) = $0.41 \times$ mass of protein $\left(\mu \mathrm{g} / \mathrm{cm}^{2}\right)^{27}$. Based on the area of tape strips and a SC density of $1 \mathrm{~g} / \mathrm{cm}^{3}$, the SC thickness or depth may be calculated using this equation, SC thickness $(\mu \mathrm{m})=$ mass of protein $\left(\mu \mathrm{g} / \mathrm{cm}^{2}\right) /\left[100 \times \mathrm{SC} \text { density }\left(\mathrm{g} / \mathrm{cm}^{3}\right)\right]^{27,28}$.

The amount of IBU in the tape strips was quantified by immersion of each tape strip in $1.5 \mathrm{~mL}$ of acetonitrile and $1 \mathrm{M}$ hydrochloric acid $(90: 10)$ for $12 \mathrm{~h}^{29}$. The extracts were subsequently analyzed using HPLC.

\section{HPLC analysis}

Samples were analysed using an Agilent 1100 Series G1311A quaternary pump (Hewlett Packard, Santa Clara, CA, US) (flow rate $=1.2 \mathrm{~mL} / \mathrm{min}$ ) with a G1315B Diode 
Array Detector set at $220 \mathrm{~nm}$. The stationary phase was a Shiseido Capcell Pak $\mathrm{C}_{18} 5$ $\mu \mathrm{m}$ column ( $250 \times 4.6 \mathrm{~mm}$ ) (Tokyo, Japan) attached to a SecurityGuard ${ }^{\mathrm{TM}}$ guard column with a $\mathrm{C}_{18}$ cartridge (Phenomenex, Macclesfield, UK). The mobile phase consists of $80 \%$ methanol, $20 \%$ water and $0.1 \%$ TFA. Samples were injected via a $25 \mu \mathrm{L}$ loop and the retention time was $5.8 \mathrm{~min}$. Calibration curves were developed based on the concentration calculated from the AUC. This HPLC method was validated according to $\mathrm{ICH}$ guideline Q2(R1) ${ }^{30}$. The limits of detection and quantification were 0.1 and 0.5 $\mu \mathrm{g} / \mathrm{mL}$, respectively.

\section{Polarized light microscopy (PLM) and scanning electron microscopy (SEM) imaging of tape strips}

The tape strips were examined for crystals that had formed by using a polarized light microscope (Nikon Microphot FXA, Tokyo, Japan). The images were captured using an INFINITY2 digital camera and INFINITY2 software (Lumenera Corp., Ontario, Canada) at $10 \times$ magnification. SEM images for the selected tapes were taken using a FEI QUANTA 200 F SEM (Eindhoven, The Netherlands). Prior to examination, samples were coated with gold using a sputter coater (Quorum Q150T, Ringmer, UK). The images were collected primarily for the tapes obtained following application of the saturated solution of IBU in neat PG as this was the formulation that was most likely to trigger drug crystallization in skin. IBU drug crystals, formed from the saturated solution of IBU in neat PG via evaporation at room temperature, were used as a reference for comparison.

\section{Localized nano-thermal analysis (nano-TA)}

Nano-TA measurements were carried out using a VESTA ${ }^{\circledR}$ Nano Thermal Analyser (Anasys Instruments Corp., Santa Barbara, CA, US) with a nano-TA probe (VITA-HENANOTA-200 Bruker AXS S.A.S, Marne la Vallee Cedex 2, France) as described previously ${ }^{10}$. In brief, the penetration of the probe into the sample surface on heating is recorded as a thermal event, measuring a local thermal transition. A TTM image is 
created by mapping an area of interest in a grid pattern with thermal transitions measured and coded using a color palette. The samples observed using TTM included IBU drug crystals from a saturated solution of IBU in neat PG, and selected tape strips collected from porcine skin following application of $P G$ and saturated solutions. The samples were stored at room temperature (relative humidity: $30-40 \%$ ) and scanned within $3-5$ days after the permeation study. The LTA measurement was obtained with a heating rate of $10^{\circ} \mathrm{C} / \mathrm{s}$ from $25^{\circ} \mathrm{C}$ to $250^{\circ} \mathrm{C}$, a cooling rate of $100^{\circ} \mathrm{C} / \mathrm{s}$ and data rate of 20 point/s. The area of the TTM images ranged from $50 \times 50 \mu \mathrm{m}, 30 \times 30 \mu \mathrm{m}$ to $10 \times 10$

$\mu \mathrm{m}$ at a resolution of $1 \times 1 \mu \mathrm{m}$. The scanning time ranged from $10-15 \mathrm{~min}$ for an area Deleted: ranges of $10 \times 10 \mu \mathrm{m}$ to $60-90 \mathrm{~min}$ for an area of $50 \times 50 \mu \mathrm{m}$.

\section{Photothermal microspectroscopy (PTMS)}

An Explorer AFM (Veeco Instruments Ltd., Santa Barbara, CA, US) fitted with a Wollaston wire thermal probe (Veeco Instruments Ltd., Santa Barbara, CA, US) was interfaced with an FTIR spectrometer (Vertex 70, Bruker Optics Ltd., Coventry, UK) for PTMS studies. The operation and set up of the technique is described in detail elsewhere ${ }^{11,12}$. A photothermal signal is collected due to the vibrational-induced temperature change of a sample upon absorption of IR radiation. These changes produce a spectrum similar to the output measured using conventional IR spectroscopy. Topographic images were first obtained for the sample of interest including tape strips from untreated PSC, PSC treated with PG and saturated solutions of IBU in neat PG. For the sample measurement, several locations of interest on the topographic images were chosen. If topographic images were not taken due to very rough surface, an area of interest was identified through the observation under polarized light microscope. The probe was in contact with the sample before spectra collection. Spectra were also collected for IBU crystals using a thermally assisted particle sampling approach ${ }^{21,31}$. Using this method, the probe selected an individual particle from a lightly scattered sample of IBU crystals on a flat surface. A background scan was carried out with the probe in air before a sample measurement. Spectra were obtained in continuous scan 
mode with a total of 1000 scans at a resolution of $8 \mathrm{~cm}^{-1}$ over a wavelength range of $4000 \mathrm{~cm}^{-1}$ to $600 \mathrm{~cm}^{-1}$.

The photothermal FTIR spectra for individual samples (untreated PSC, PSC treated with PG and IBU crystals) were compared to the ATR-FTIR spectra measured using the same FTIR spectrophotometer but equipped with a DigiTect ${ }^{\mathrm{TM}}$ detector and a GoldenGate $^{\mathrm{TM}}$ single-reflection diamond ATR accessory (Specac Ltd., Orpington, UK).

For PSC treated with PG, the hydrated PSC was fully immersed in an excess amount of Deleted: excessive $\mathrm{PG}$ for $24 \mathrm{~h}$ at room temperature and dried by pressing between 2 pieces of tissue paper repeatedly until the sheets did not wet the paper further ${ }^{23,32}$. Each sample was run for 64 scans with a resolution of $2 \mathrm{~cm}^{-1}$ over a wavelength range of $4000 \mathrm{~cm}^{-1}$ to 600 $\mathrm{cm}^{-1}$.

Spectra from both PTMS and ATR-FTIR spectroscopic measurement were analyzed using Opus software version 6.0 (Bruker Optics Ltd., Coventry, UK) and OriginPro 9.0.0 (OriginLab Corp., Northampton, MA, US).

\section{Data analysis and statistics}

All statistical tests were performed using SPSS version 21 (IBM Corp., Armonk, NY, US) and $p<0.05$ was chosen as the minimum level of significance in all cases. For data analysis, the Mann-Whitney test was chosen because the results were accepted as non-parametric data, showing a normal distribution with the skewness and kurtosis value more than 1 (or less than -1). Results are expressed as means with standard deviation (SD).

\section{RESULTS}

\section{Solubility studies of saturated solution of IBU}

The saturated solubility of IBU in mixtures of $P G$ and water at $32^{\circ} \mathrm{C}$ increases exponentially with increasing PG proportion (data not shown) as reported by Watkinson, et al. ${ }^{33}$ and Manrique, et al. ${ }^{34}$. The saturated solubility of IBU was $289.56 \pm 3.76 \mathrm{mg} / \mathrm{mL}$ 
in neat PG, and the values for PG:water (75:25, 50:50, and 25:75), were $34.43 \pm 0.43$ $\mathrm{mg} / \mathrm{mL}, 3.57 \pm 0.40 \mathrm{mg} / \mathrm{mL}$ and $0.54 \pm 0.04 \mathrm{mg} / \mathrm{mL}$ respectively. lervolino, et al. ${ }^{35}$ reported similar results for $I B U$ in $P G$ at $32^{\circ} \mathrm{C}(301.3 \mathrm{mg} / \mathrm{mL})$. For the saturated solution of IBU in water the solubility was $0.14 \pm 0.01 \mathrm{mg} / \mathrm{mL}$.

\section{Permeation of IBU in porcine ear skin}

Figure 1, shows the permeation profiles of IBU in porcine skin following application of Deleted: Figure 1 saturated solutions of IBU in neat PG, water and PG:water (75:25, 50:50). The data for the permeation profiles using the saturated solution of IBU in PG:water (25:75) and water only are not reported as the concentrations of IBU fell below the limit of HPLC quantification $(0.5 \mu \mathrm{g} / \mathrm{mL})$. The permeation of IBU from a finite dose $\left(2 \mu \mathrm{L} / \mathrm{cm}^{2}\right)$ increased with increasing PG proportion. The cumulative amount of IBU achieved after $24 \mathrm{~h}$ permeation was found to be the highest for neat $P G\left(282.5 \pm 63.4 \mu \mathrm{g} / \mathrm{cm}^{2}\right)$, followed by PG:water (75:25) with a value of $38.4 \pm 9.1 \mu \mathrm{g} / \mathrm{cm}^{2}$ and PG:water (50:50) with a value of $7.0 \pm 0.5 \mu \mathrm{g} / \mathrm{cm}^{2}$. Enhanced drug penetration appears to reflect the influence of $P G$ on the solubility and partitioning behavior of IBU ${ }^{33}$.

Initially, the permeation of IBU increased linearly but exhibited a plateau over time. Depletion of $P G$, is likely to be the major factor affecting the permeation of IBU across porcine skin ${ }^{36,37}$. PG crosses the skin barrier more quickly than the drug and this will result in solvent loss from the formulation applied ${ }^{36}$. Also, solvent evaporation is expected to limit the ability of $P G$ in enhancing permeation of IBU ${ }^{37,38}$. With reference to the thermodynamic activity of the formulation, solvent depletion would also be expected to increase the possibility of drug crystallization in and on the skin. 


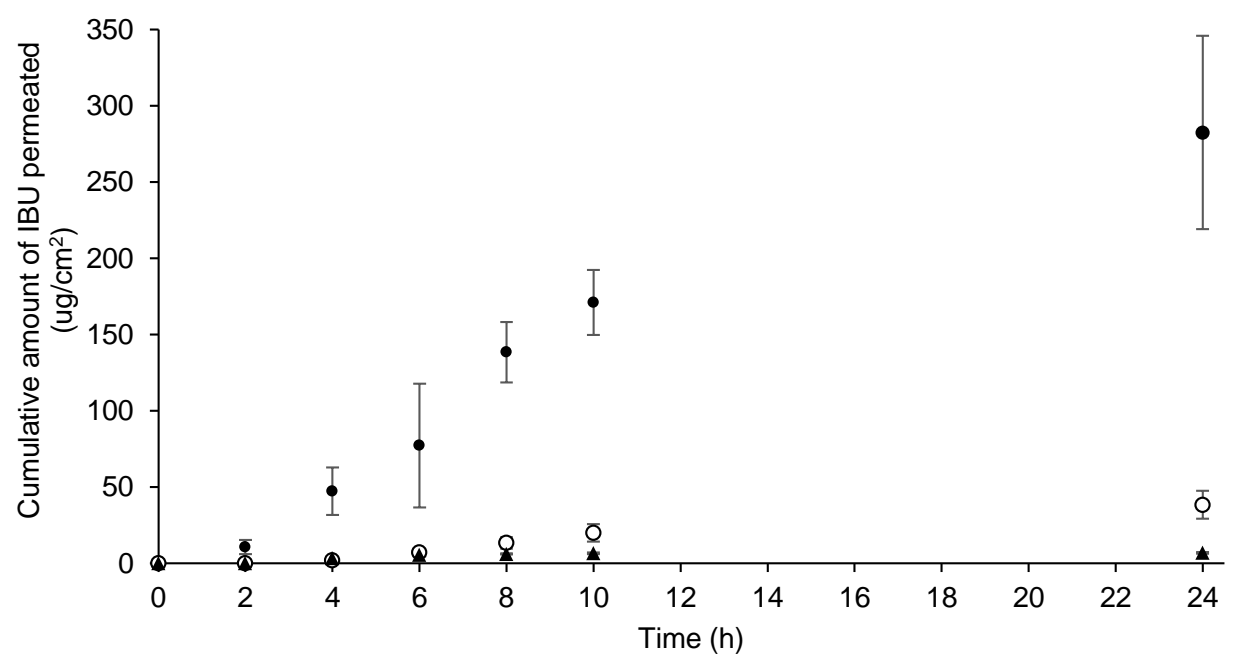

Figure 1 Permeation profiles of IBU in porcine skin for saturated solutions of IBU in neat PG $(\bullet)$ and $P G: w a t e r(75: 25(\circ), 50: 50(\Delta))(n=5$, mean \pm SD)

\section{In vitro tape stripping and protein content quantification using IR densitometry}

IR densitometry was applied to quantify the protein content of SC on the tape strips.

This method provides a consistent result in the estimation of SC thickness ${ }^{27,39}$. In this study, the results obtained from IR densitometry were compared for the saturated solutions of IBU in different mixtures of PG:water and PG alone with the untreated PSC. The total SC thickness removed for 20 sequential tape stripping after application of the | various preparations is shown in Table 1 , The only preparation that resulted in Deleted: Table 1 significantly lower SC thickness removed from the control was the saturated solution of IBU in neat PG (Mann-Whitney test, $p<0.05$ ). 
Table 1 The estimated thickness of PSC removed with 20 tape strips following application of different preparations $(n=5$, mean \pm SD)

\begin{tabular}{cc}
\hline Preparation & SC thickness removed $(\mu \mathrm{m})$ \\
\hline Control & $6.6 \pm 0.8$ \\
PG only & $6.6 \pm 0.7$ \\
Saturated solution of IBU in neat PG & $4.4 \pm 0.9^{*}$ \\
Saturated solution of IBU in PG:water (75:25) & $6.1 \pm 0.5$ \\
Saturated solution of IBU in PG:water (50:50) & $6.7 \pm 1.0$ \\
Saturated solution of IBU in PG:water (25:75) & $6.8 \pm 0.4$ \\
Saturated solution of IBU in water & $5.7 \pm 3.3$ \\
\hline
\end{tabular}

* indicates statistically significant difference (Mann-Whitney test, $p<0.05$ )

Figure 2 shows the comparison of the amount of SC protein removed from the 20

Deleted: Figure 2 sequential tape strips for control (untreated) and treated porcine skin with PG alone and various saturated solutions of IBU. Following the application of $P G$ alone, the amount of the removed protein showed no statistical differences (Mann-Whitney test, $p>0.05$ ) compared with the control with exception of the first tape $(p<0.05)$ (Figure $2 A)$. The SC collected from the first tape strip has the highest exposure time to PG before PG evaporates or permeates through the skin. $P G$ had no influence on the removal of the $\mathrm{SC}$ in the deeper layers.

With the application of a saturated solution of IBU in neat PG, the amount of the SC protein removed was significantly affected by the formulation compared with the control up to the tenth removed tape $(p<0.05)$ (Figure $2 \mathrm{~B})$. The removal of SC protein from the subsequent tape strips was not influenced by the formulation applied.

A similar trend was observed for the saturated solution of IBU in PG:water (75:25, 50:50) and 25:75). However, the effects of these solutions on the amount of the SC protein removed was less marked in the deeper layers of the skin as compared with the control 
as the proportion of $P G$ reduces as shown in Figure $2 \mathrm{C}-\mathrm{E}(p<0.05)$. Without the presence of $P G$, there is no significant difference in the amount of the SC protein removed from all the tapes following the application of the saturated solution of IBU in water and the control (Figure $2 \mathrm{~F}$ ).

Overall, $P G$ alone (Figure $2 A$ ) has only a minimal impact on the amount of SC protein removed except for the first tape strip. However, with the application of IBU formulations, the removal of SC protein was significantly lower than that for the PG alone. The reduction in the SC protein removed for these formulations may be explained by the presence of drug in the form of crystals in the skin which hindered the removal of SC protein. The drug crystals sandwiched between the tape and SC may prohibit the further adhesion of SC to the tape. The decrease of SC protein removal is more significant when saturated solutions of IBU with higher proportions of $P G$ were applied especially in neat PG. This could be related to the solubility of IBU in the formulations. High drug solubility would indicate a greater potential for drug to recrystallize from the formulations. This may result in difficulty in removal of SC protein. For saturated solutions of IBU where IBU was not as soluble such as PG:water (50:50, 25:75) and water only, the formation of crystals is less likely in the SC. This should result in minimal differences in $\mathrm{SC}$ protein content removed after the first tape stripping of the tissue.
Deleted: Figure 2

Deleted: Figure 2

Deleted: Figure 2

Deleted: saved

Deleted: penetration enhancer (

Deleted: )

Deleted: very

Deleted: no 

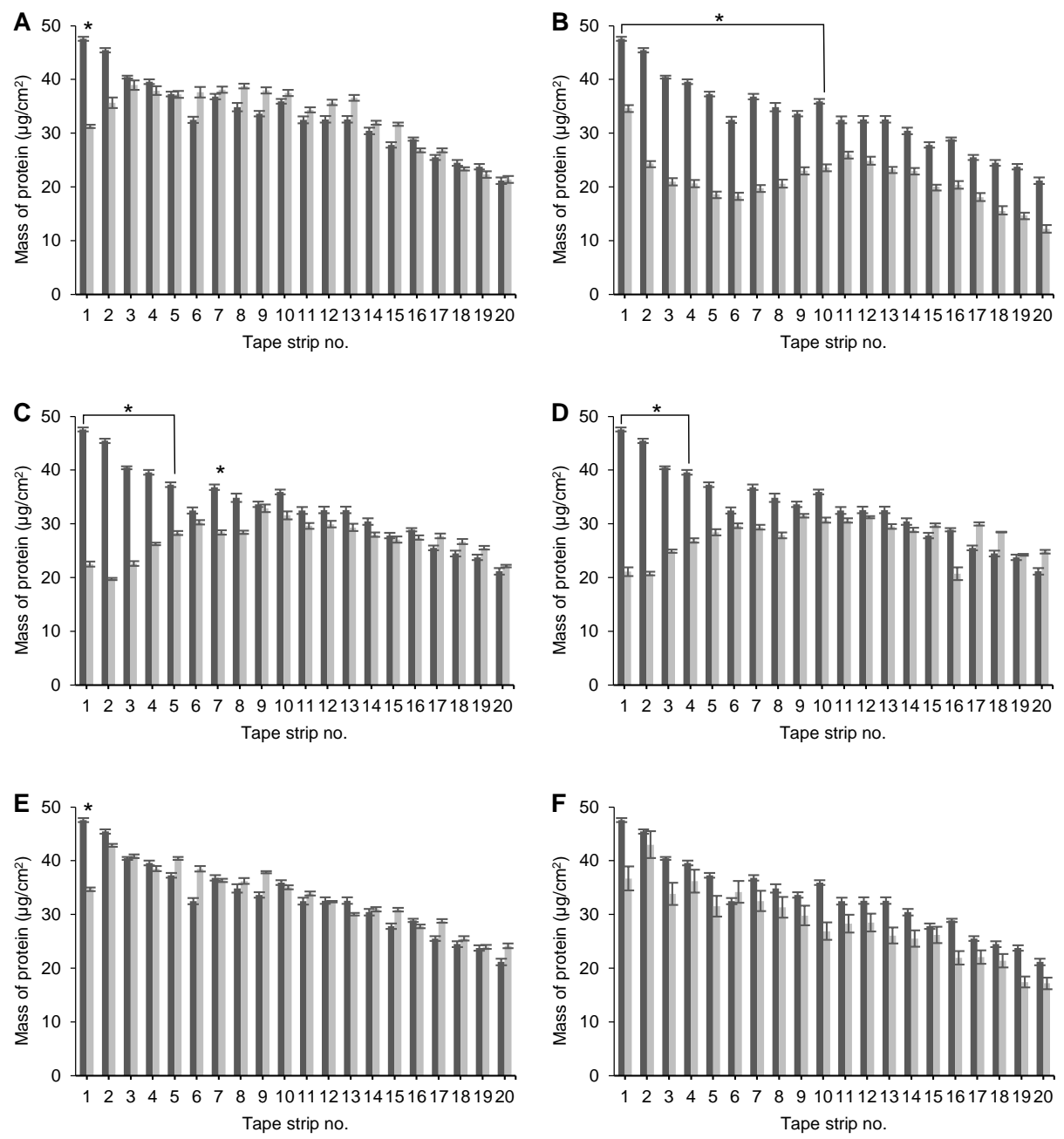

Figure 2 Influence of the applied saturated solution on the amount of removed PSC proteins in vitro. The dark grey bars, represent the amount of SC protein removed from untreated porcine skin (control). The light grey bars represent the amount of SC protein removed after $24 \mathrm{~h}$ application of (A) PG, (B) saturated solutions of IBU in neat PG, (C) PG:water (75:25), (D) PG:water (50:50), (E) PG:water (25:75) and (F) water ( $n=5$, mean \pm SD). * indicates statistically significant difference (Mann-Whitney test, $p<0.05$ ). 


\section{Extraction of IBU from the tape strips}

The amounts of IBU in the SC following application of saturated solutions in PG:water (25:75) and water were very low and could not be quantified using HPLC as they fell below the limit of quantification $(0.5 \mu \mathrm{g} / \mathrm{mL}$ ). Data (not shown), thus, were not considered from these vehicles. The IBU extracted from the tapes across the SC could only be quantified for the saturated solutions in neat PG and PG:water $(75: 25,50: 50)$ as shown in Figure 3 ,

Deleted: Figure 3

After application of the saturated solution of IBU in neat PG, the amount of IBU in the $\mathrm{SC}$ was relatively high in the first tenth tape strip as compared with the rest of the tapes. For saturated solutions in PG:water (75:25) and (50:50), a very small amount of IBU was detected in the third and the first tape strips, respectively.

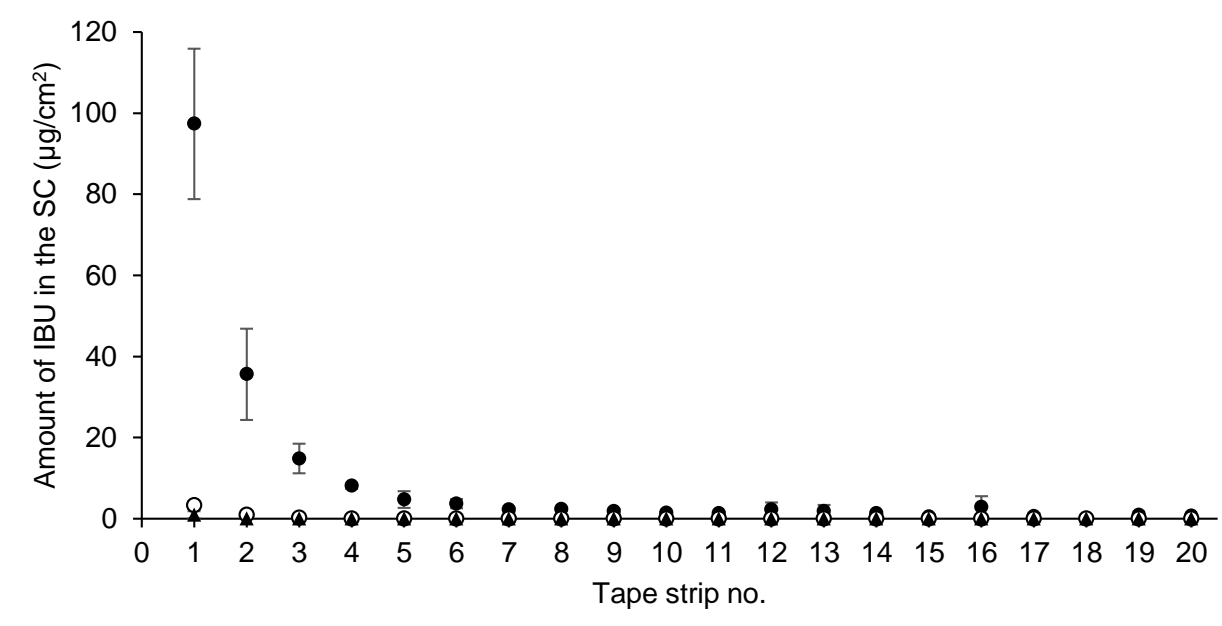

Figure 3 Amount of IBU in the SC after $24 \mathrm{~h}$ in vitro permeation of saturated solutions in neat PG $(\bullet)$ and PG:water $(75: 25(\circ), 50: 50(\triangle))(n=4$, mean \pm SD) 


\section{Microscopic imaging of tapes - PLM and SEM}

Microscopic observation using PLM was conducted for the tape strips collected after the $24 \mathrm{~h}$ permeation studies with different saturated solutions. Selected PLM images are shown in Figure 4A - E. Flat plate-shaped crystals were observed randomly in PSC which were similar to the IBU crystals formed following solvent evaporation from the saturated solution of IBU in neat PG at room temperature. Drug crystal formation in the $\mathrm{SC}$ is represented by the crystalline spherulites in the seventh tape following application of the saturated solution of IBU in PG: water (50:50) (Figure $4 \mathrm{C}$ ). This observation suggests that crystals may grow in a radial pattern in the SC.

The presence of crystal-like structures was also observed with SEM for several tape strips obtained after the $24 \mathrm{~h}$ permeation studies for the saturated solution of IBU in neat PG (Figure 4F). These SEM images were compared with those obtained from untreated PSC (not shown) showing only a flat surface. Crystal protrusions detected on the first tape strip in Figure 4F may be a result of the attachment of the crystals formed on the surface of the skin which then project through the tissue.

Deleted: Figure 4

Deleted: Figure 4

Deleted: 

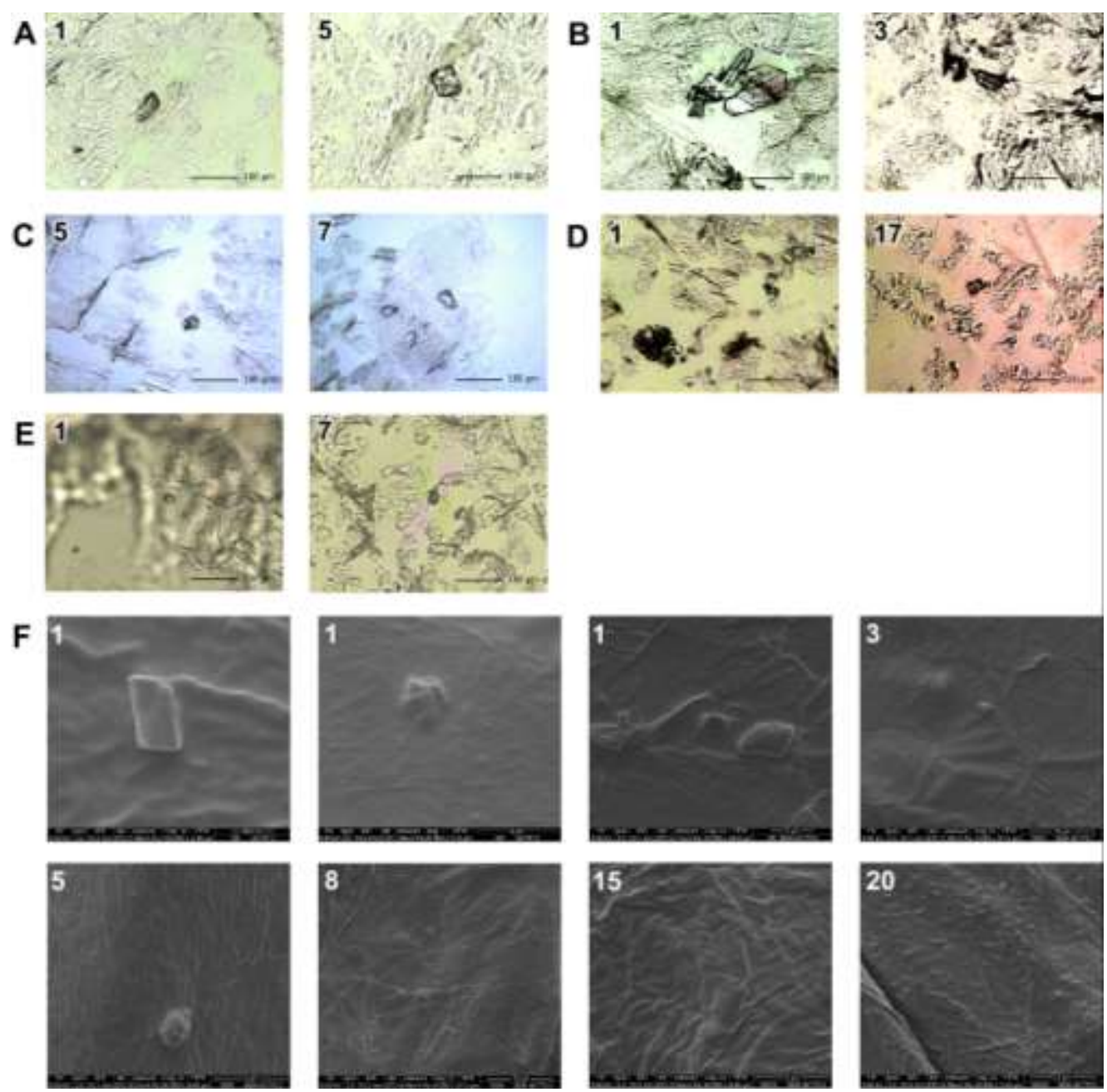

Figure 4 (A - E) PLM and (F) SEM images of IBU crystals observed on the selected tapes following application of saturated solutions of IBU in (A and $F$ ) neat PG, (B) PG:water (75:25), (C) PG:water (50:50), (D) PG:water (25:75) and (E) water. The number in the microscopic images refers to the tape strip number. 


\section{Localized nano-TA}

\section{IBU drug crystals}

IBU crystals formed following evaporation of a saturated solution of IBU in neat PG were used for the TTM study. A flat surface of an IBU crystal was selected for the TTM image with the assistance of the optical microscope as illustrated in Figure 5 with a dimension of $10 \times 10 \mu \mathrm{m}(1 \times 1 \mu \mathrm{m}$ detection resolution). This image shows a homogenous color distribution (blue) with a transition temperature around $55^{\circ} \mathrm{C}$. This temperature is slightly lower than the melting point of IBU measured using DSC, recorded at $\sim 79^{\circ} \mathrm{C}$ (DSC thermograms not shown).

The modification of the crystals' size and melting of crystal surface molecules would be expected to result in a notable drop in the melting temperature in comparison with the bulk crystals. Previously, crystals in the nanometer range or nanocrystals were reported to have lower melting temperatures than the bulk crystalline form ${ }^{40-42}$. This sizedependent melting temperature and entropy depression were also reported in organic crystals because of the difference of vibrational spectrum of the surface region as compared to the bulk ${ }^{43,44}$. The size of the crystals is expressed as the ratio between the percentage of surface molecules and the interior molecules. The reduction in this ratio as the crystals' size decreases is reflected by a drop in the vibrational entropy of the crystals. Apart from the size-dependent melting depression, this melting reduction is also related to the shape of the crystals owing to a large ratio of the number of surfaceto-volume atoms ${ }^{45}$. Therefore, the melting involving only the surface molecules of drug crystals could also be a major factor causing a lower transition temperature in the TTM data.
Deleted: Figure 5

Deleted: recording

Deleted: remarkable 

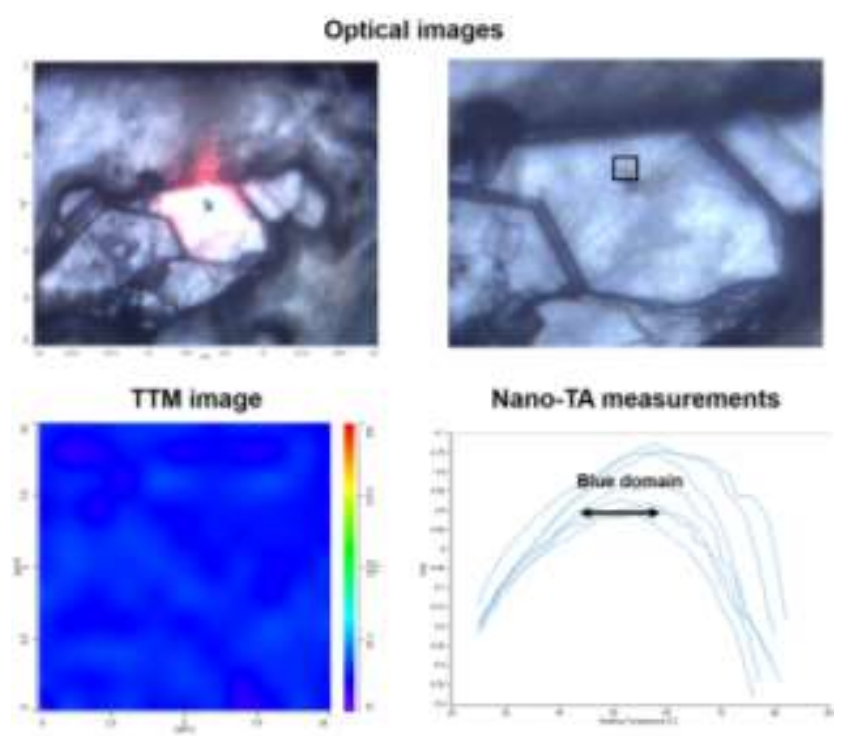

Figure 5 TTM data for the IBU crystals formed from the saturated solution of IBU in neat PG (area: $10 \times 10 \mu \mathrm{m}$; resolution: $1 \times 1 \mu \mathrm{m}$ )

\section{PSC with application of a saturated solution of IBU in neat PG}

The highest permeation of IBU and the greatest IBU content in the SC respectively, were observed after the application of the saturated solution of IBU in neat PG. This also indicates a higher amount of drug deposited in the SC. Considering these factors, the results here focus only on the TTM measurement from this formulation.

After the application of this preparation, the TTM image of the first tape showed four major domains: red (yellow), purple, blue and green colors (Figure 6A). The transition temperature around $220^{\circ} \mathrm{C}$ within the red (yellow) domain refers to the SC while the adhesive side of the tape shows a purple domain $\left(25^{\circ} \mathrm{C}\right)$. The green domain was assigned to the PG-affected SC as reported previously ${ }^{10}$. PG weakened the biomechanical properties of the SC by extracting water from the SC protein. The weakened SC layer could not resist the penetration force of the heated probe tip, resulting in the lower thermal transition and the generation of green domains. 
The presence of IBU crystals on the tape is evident from the appearance of blue domains in this TTM image. The blue regions were scattered in the first tape obtained following application of a saturated solution of IBU in neat PG (Figure 6A). This random scattering of the blue regions also supports the presence of IBU crystals on the tape.

The green domain has been previously assigned to the PG-treated PSC. However, this domain has a transition temperature range slightly lower than that of PSC influenced by $P G$, represented here by a more intense green color. Apart from the transition depression by PG on PSC, it is reasonable to suppose that the IBU crystals have some prominent effects on the transition temperature of SC, emerging as a greener domain in the TTM image. From previous observations using SEM (Figure 4F), the crystals attached to the tape may be overlaid by a layer of corneocytes. The tape was inverted for scanning under the thermal probe and this probe was in contact with the crystals indirectly. Owing to the higher transition temperature of PSC, it is proposed here that a slower heat transfer may occur from the probe to the crystals through this SC layer. Initially, =when the probe heated up the $\mathrm{PSC}_{p}$ the crystals beneath the SC begin to melt, This, therefore, would result in an earlier penetration of the probe as the melting of the crystals would occurr, faster than that of the SC (Figure S1 in the Supporting Information). Subsequently, a lower transition temperature within the green domain was observed. In some regions showing the red (yellow) color, there may also exist many keratin filaments which would minimize or prevent the heat transfer to the crystals underneath.

Although a specific blue region could not be precisely mapped in the TTM image, it is interesting to note the existence of double transitions in some regions, reflecting the possibility of formation of very small drug crystals or nanocrystals. The double transitions consisted of both transition peaks recorded in blue and green domains but only the latter peak is present in the TTM image (Figure 6B). It is therefore shown in the TTM images as a green domain. IBU may crystallize in the inter-corneocyte space ( 75 $\mathrm{nm}$ ) as nanocrystals when the solvent mixture depletes from the skin surface mainly via evaporation. The formation of nanocrystals in the inter-corneocyte space would enable the penetration of the thermal AFM probe through these crystals once they melt, reaching the SC layers beneath. Further heating results in the formation of a second
Deleted: Figure 6

Deleted: Figure 4

\begin{tabular}{|l|}
\hline Deleted: At \\
\hline Deleted: the time \\
\hline Deleted: to a certain extent \\
\hline Deleted: were just starting \\
\hline Deleted: soften \\
\hline Deleted: ed \\
\hline Deleted: softening \\
\hline Deleted: Detentually \\
\hline Deleted: ed \\
\hline Deleted: perfectly \\
\hline Deleted: Figure 6 \\
\hline
\end{tabular}


transition peak in the green domain. This mechanism of generation of the double transitions is illustrated in Figure S2 in the Supporting Information. Double transitions may also appear as red (yellow) domains when the probe approaches the SC with high keratin content. However, most of the double transitions appeared as green domains in this case. These green domains are likely related to the dehydration effect of PG on the SC protein,

On observing crystals under a PLM, a number of tapes with crystals (tape number 3, 5, 8, 15 and 20) were selected for nano-TA, allowing the depth profiling of the crystallization process across PSC (Figure 7). These TTM images contained the same color domains (red, green and purple) as observed previously, for the first tape obtained following application of the same saturated solution. Blue domains and double transitions were also detected in these TTM images, strongly suggesting the presence of crystals in the SC. The appearance of blue domains and double transitions were infrequently identified in the TTM images from the tapes in the deeper layer of the SC. This observation is in line with the microscopic images obtained.

A

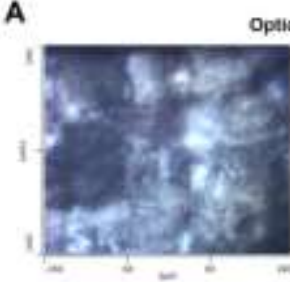

TTM image

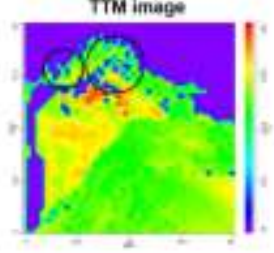

Optical images

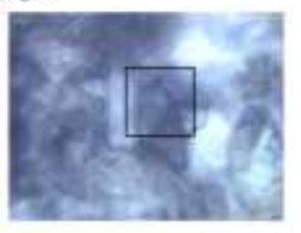

Nano-TA measurements

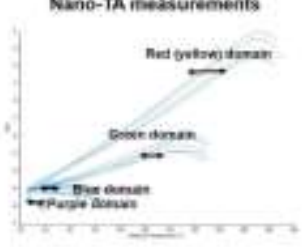

B

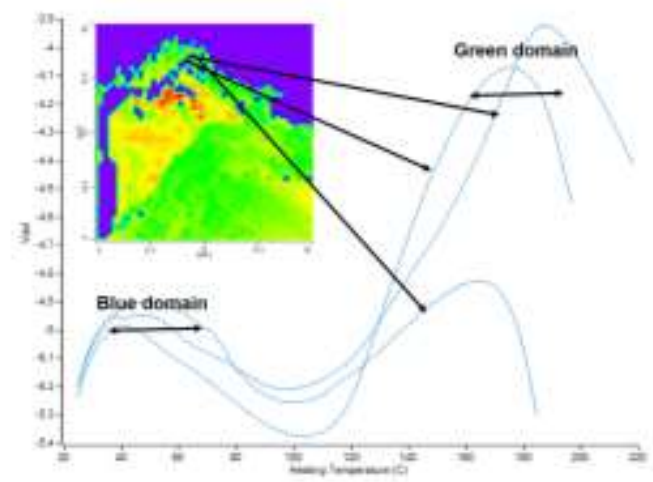

Deleted: by PG

Deleted: Figure 7

Figure 6 (A) TTM data and (B) the double transitions recorded in the first tape removed after application of the saturated solution of IBU in neat PG (area: $50 \times$ $50 \mu \mathrm{m}$; resolution: $1 \times 1 \mu \mathrm{m})$. The blue domains are present in the circles of the TTM image in (A). 

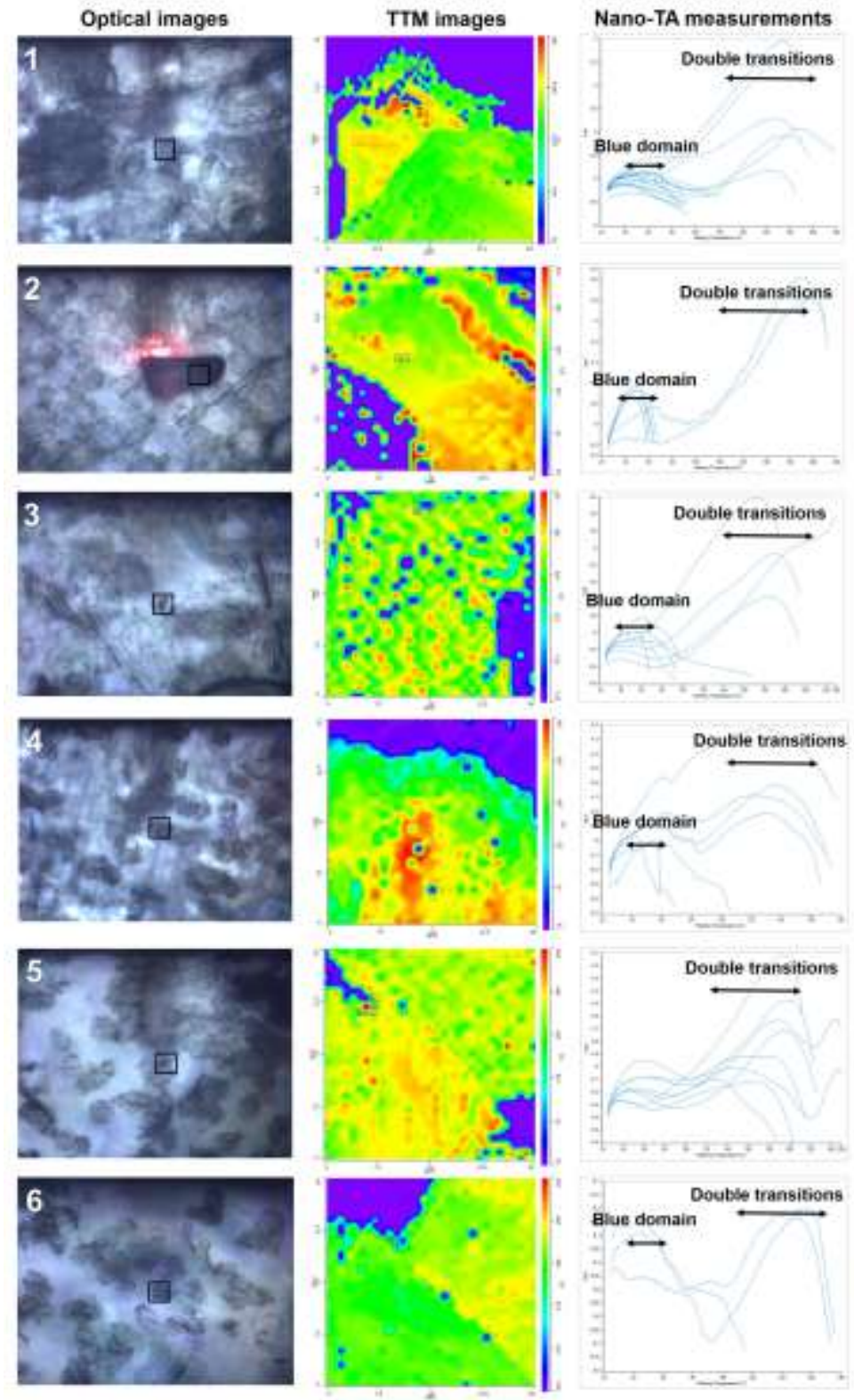

Figure 7 TTM data showing the presence of blue domains and double transitions (pixels denoted with [+]) in the tapes removed after application of the saturated solution of IBU in neat PG (area: $50 \times 50 \mu \mathrm{m}$ (first tape), $30 \times 30 \mu \mathrm{m}$ (third twentieth tapes); resolution: $1 \times 1 \mu \mathrm{m}$ ). The number in the optical images refers to the tape strip number. 


\section{PTMS}

The presence of drug crystals in the SC was also confirmed using PTMS apart from the determination of its local thermal transition measured by TTM. This photothermal technique generates a spectrum similar to that obtained in conventional FTIR spectroscopy. In this study, the photothermal FTIR spectra for the individual materials, namely untreated PSC, PSC treated with PG and IBU crystals were found to be comparable to the corresponding ATR-FTIR spectra (Figure S3 in the Supporting Information).

The photothermal FTIR spectra for the first tape strip removed from untreated PSC and PSC treated with PG showed no major differences (Figure S4 in the Supporting Information). However, the protein-related vibrations for PG-treated SC were found to be greatly reduced in intensity, including amide $A$ and $B$ peaks at the $3500-3000 \mathrm{~cm}^{-1}$ region as well as amide I and II peaks at $1700-1500 \mathrm{~cm}^{-1}$ region. This could be the dehydration effect of PG on the SC protein ${ }^{10}$.

After applying the saturated solution of IBU in neat PG, no AFM image was obtained because the loosely-attached drug crystals on the tape hindered accurate recording, Therefore, photothermal FTIR spectra were collected from the area where crystal-like structures on the tape were confirmed using PLM. These photothermal FTIR spectra and the respective scanning sites are shown in Figure 8. Clearly, the presence of the drug crystals is indicated in the photothermal FTIR spectra (highlighted in the box of Figure 8All and $\mathrm{BII}$ ) of $\mathrm{IBU}$ with the presence of its characteristic $\mathrm{C}=\mathrm{O}$ stretching vibrations $\left(1720 \mathrm{~cm}^{-1}\right)$. However, when the experiment was repeated on the second tape strip, the photothermal FTIR spectra (not shown) obtained were only from the SC. Although crystal-like structures were observed under the microscope, the overlaying SC may prevent the detection of IBU crystals using PTMS. In addition, the fact that no drug was detected in the second tape strip may be due to the limitation in sensitivity of the Wollaston wire thermal probe. The exposed V-shaped probe tip with a contact radius of $\sim 1 \mu \mathrm{m}$ may be too large, to detect the comparatively small IBU crystals found in the deeper layers of the skin. Therefore, the subsequent tape strips were not examined with PTMS. 

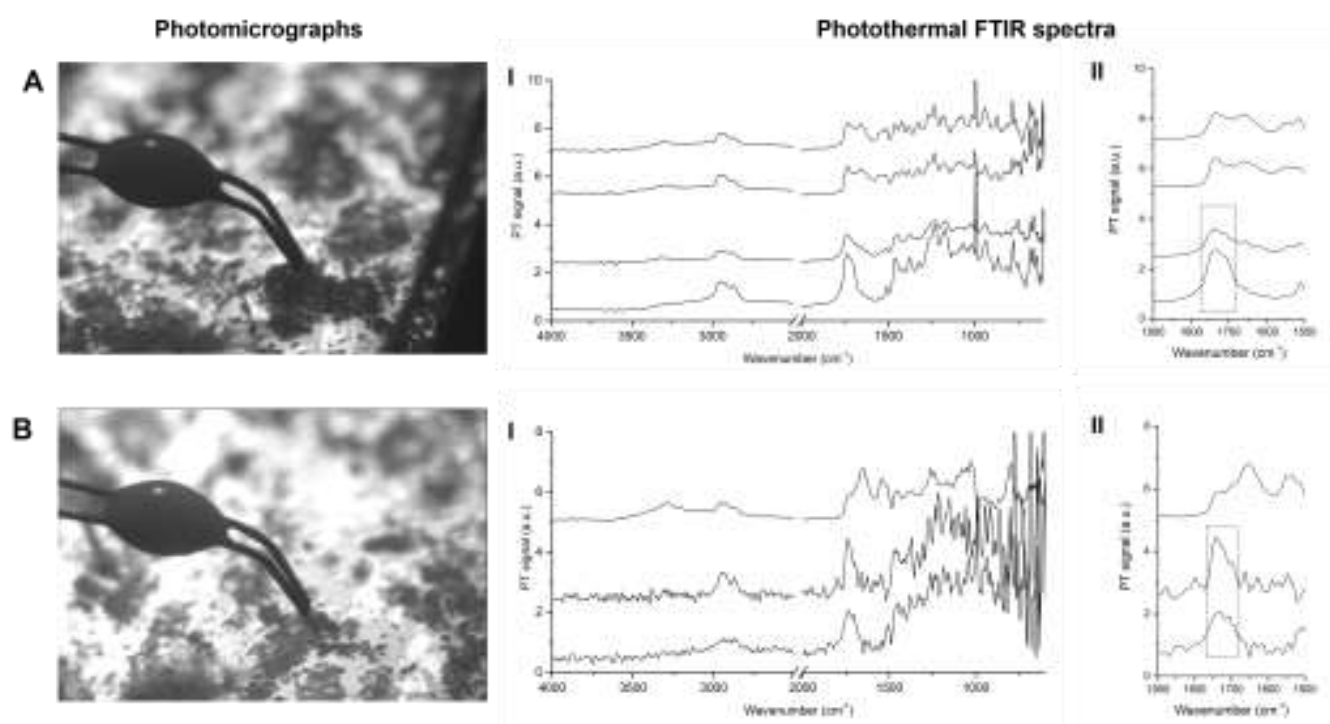

Figure 8 Photomicrographs and corresponding photothermal FTIR spectra (sites $A$ and $B$ ) of the first tape strip removed after application of the saturated solution of IBU in neat PG (I: full spectra; II: $1900-1500 \mathrm{~cm}^{-1}$ )

\section{DISCUSSION}

Drug crystallization in the SC has been identified as a major issue in topical drug delivery. In this study, localized nano-TA and PTMS were employed to examine the behavior of drug crystallization in porcine skin following topical application of various preparations with high drug thermodynamic activity.

Drug crystallization has been suggested to be a critical obstacle in penetration of drug into the skin. From the permeation studies reported here, a gradual decrease in the cumulative amount of IBU collected from the receptor phase was observed, indicating depletion of the vehicle by the end of the permeation study. We also believe that drug crystallization could be a major contribution to this observation. Drug crystallization occurring on and in a silicone membrane and human skin was previously proposed as a

\begin{tabular}{|l|}
\hline Deleted: another main cause \\
\hline Deleted: for
\end{tabular}
major limitation to effective permeation enhancement of oxybutynin and fentanyl using supersaturation ${ }^{5,6}$. Klang, et al. ${ }^{46}$ suggested the possibility of drug crystallization, when 
comparing in vitro and in vivo tape stripping to validate the porcine ear as a skin permeation model. The authors proposed that drug crystals formed due to the evaporation of volatile compounds in the formulation hindered further penetration of drug.

In the tape stripping work in this study, the protein content was found to be substantially reduced after the application of a saturated solution of IBU in neat PG and PG:water (75:25, 50:50). The protein content removed decreased with increasing number of tape strips. The influence of the vehicle, $\mathrm{PG}$, on the $\mathrm{SC}$ protein removal has been ruled out except for the first tape strip. With this, we believe that the reduction in the SC protein removed may be due to the presence of drug. Even though drug extraction results confirmed the presence of IBU in the SC, this method of analysis does not differentiate the drug in the solution or crystalline form. However, microscopic images (PLM and SEM) showed the presence of crystal-like structures on the tape strips together with corneocytes. Thus, it is speculated that the existence of drug in the crystalline form in the SC may interfere with the adhesive film's ability to extract more corneocytes, especially in the first few SC layers.

The presence of the crystalline formof IBU is further confirmed using two probe-based microscopic approaches. Localized nano-TA using TTM enables the detection ofsubstances at the nano scale. Nano-TA analysis on tape strips collected after permeation studies jdentified a number of important events which provide the evidence of IBU presence as the crystalline form. The transition temperature of the IBU crystals at about $55^{\circ} \mathrm{C}$ (blue domain) is direct evidence for the presence of IBU crystals. In addition, IBU crystals were detected from the transition depression that resulted from the delay of heat transfer from the probe to the drug crystals sandwiched between PSC and the tape. Also, the first transition in the double transitions represents the melting of IBU crystals. This provides further evidence for drug crystal formation in the skin, most probably as very fine crystals. The TTM data support the observations recorded in PLM and SEM images where drug crystals were observed randomly on the tape strips. Even though HPLC analysis could not quantify the drug extracted from the tapes in the deeper layers of the SC, the presence of crystals, formed, possibly in the inter-corneocyte spaces, could be detected with nano-TA. 
Because the highest drug concentration and permeation was obtained with neat PG, the appearance of blue domains and double transitions was evident in the TTM images from most of the tape strips collected for this experiment. However, these thermal transitions are fewer in the deeper layers of the skin as indicated by the number of tape strips. These observations were also less evident with the co-solvent system (PG and water) or water (data not shown). These results may reflect the lower incidence of drug crystallization from those preparations with a lower drug concentration.

To further confirm the detection of drug crystals on the tapes, another probe-based spectroscopic approach using PTMS was employed. Several single point scans of PTMS on the tape strips after application of the saturated solution of IBU in neat PG mimicked the photothermal FTIR spectra of IBU with a strong characteristic $\mathrm{C}=\mathrm{O}$ stretching vibrations ay $1720 \mathrm{~cm}^{-1}$. This is in good agreement with the nano-TA of skin samples with drug crystals using TTM. Considering the larger probe used in PTMS compared with TTM, the possibilities for probing into the deeper layers of the skin to assess fine drug crystal formation were limited.

With the application of these two probe-based microscopies, this study provides new insights into the phenomenon of drug crystallization in the SC in vitro. The visualisation of drug crystals was achieved here through direct and indirect melting of drug crystals on and beneath the SC layers at the submicron or nano scale. This was then complemented by the local spectroscopic information obtained. More importantly, these techniques are able to elucidate the location and position of drug crystals in the SC through the unique thermal events including double transitions and transition depression. We believe that this is an important and novel application of these analytical approaches, and the findings would not have been observed with conventional microscopic techniques, including Raman microscopy. The results reported here also provides further insights into our understanding of drug crystallization in the SC, specifically in the inter-corneocyte space of the SC.
Deleted: of

Deleted:

Deleted: a

Deleted: are

Deleted: positive gain

Deleted: which may not be able to be achieved using

Deleted: es

Deleted: This observation

Deleted: an in

Deleted: -depth

Deleted: on the behavior 


\section{CONCLUSIONS}

Overall, thermal and spectroscopic probe-based microscopic methods were successfully applied to examine the formation of IBU crystals in SC collected from tape stripping after application of a saturated solution of IBU in neat PG. Analysis of the protein removed indicated the presence of drug crystals in the SC, especially close to the skin surface. The detection of IBU crystals was confirmed by two important thermal events - melting of crystals (blue domains) and double transitions (melting of crystals and protein denaturation/degradation of PSC). PTMS results demonstrated that the crystal-like structures observed with an optical microscope were IBU crystals. These two thermal events were observed up to tape strip number 20 , which is equivalent to a depth of $\sim 4-7 \mu \mathrm{m}$ in the SC. The findings of the study provide evidence of drug crystallization in the SC in vitro.

To our knowledge this is the first use of these techniques in the field and confirms that probe-based microscopy is a sensitive technique for jnvestigating drug disposition in the skin. The number of tape strips and the use of finite dosing limit the detection of drug crystals in the deeper layers of the skin. Nevertheless, the results presented here confirm "stranding" of drug in the inter-corneocyte space of the SC most probably as very fine crystals. Future work will investigate if the methodologies reported here may be used to examine the phenomenon of crystallization of other drugs in the skin.

\section{ASSOCIATED CONTENT}

\section{Supporting information}

Figure S1 and S2 show the proposed mechanisms of the creation of the green domain and double transitions in TTM measurements.

Figure S3 and S4 show the PTMS measurements for untreated PSC, PSC treated with $P G$ and IBU crystals. 


\section{AUTHOR INFORMATION}

\section{Corresponding author}

`Email: choonfugoh@usm.my / gohchoonfu@hotmail.com. Phone: +604-6532074. Fax: $+604-6570017$.

\section{ORCID}

Choon Fu Goh: 0000-0001-5084-4074

\section{Notes}

The authors declare no competing financial interest.

\section{ACKNOWLEDGEMENTS}

The authors thank Ministry of Education Malaysia and Universiti Sains Malaysia for PhD funding of Choon Fu Goh.

\section{REFERENCES}

1. Higuchi, T. Physical chemical analysis of percutaneous absorption process from creams and ointments. Journal of the Society of Cosmetic Chemists 1960, 11, (2), 8597.

2. Raghavan, S. L.; Kiepfer, B.; Davis, A. F.; Kazarian, S. G.; Hadgraft, J. Membrane transport of hydrocortisone acetate from supersaturated solutions: The role of polymers. International Journal of Pharmaceutics 2001, 221, (1-2), 95-105.

3. Raghavan, S. L.; Trividic, A.; Davis, A. F.; Hadgraft, J. Crystallization of hydrocortisone acetate: Influence of polymers. International Journal of Pharmaceutics 2001, 212, (2), 213-221.

4. Raghavan, S. L.; Trividic, A.; Davis, A. F.; Hadgraft, J. Effect of cellulose polymers on supersaturation and in vitro membrane transport of hydrocortisone acetate. International Journal of Pharmaceutics 2000, 193, (2), 231-237.

5. Santos, P.; Watkinson, A. C.; Hadgraft, J.; Lane, M. E. Enhanced permeation of fentanyl from supersaturated solutions in a model membrane. International Journal of Pharmaceutics 2011, 407, (1-2), 72-77. 
6. Santos, P.; Watkinson, A. C.; Hadgraft, J.; Lane, M. E. Oxybutynin permeation in skin: The influence of drug and solvent activity. International Journal of Pharmaceutics 2010, 384, (1-2), 67-72.

7. Goh, C. F.; Craig, D. Q. M.; Hadgraft, J.; Lane, M. E. The application of ATRFTIR spectroscopy and multivariate data analysis to study drug crystallisation in the stratum corneum. European Journal of Pharmaceutics and Biopharmaceutics 2017, 111, 16-25.

8. Turner, Y. T. A.; Roberts, C. J.; Davies, M. C. Scanning probe microscopy in the field of drug delivery. Advanced Drug Delivery Reviews 2007, 59, (14), 1453-1473.

9. Dai, X.; Moffat, J. G.; Wood, J.; Reading, M. Thermal scanning probe microscopy in the development of pharmaceuticals. Advanced Drug Delivery Reviews 2012, 64, (5), 449-460.

10. Goh, C. F.; Moffat, J. G.; Craig, D. Q. M.; Hadgraft, J.; Lane, M. E. Nano-thermal imaging of the stratum corneum and its potential use for understanding of the mechanism of skin penetration enhancer. Thermochimica Acta 2017, 655, 278-283.

11. Hammiche, A.; Pollock, H. M.; Reading, M.; Claybourn, M.; Turner, P. H.; Jewkes, K. Photothermal FT-IR spectroscopy: A step towards FT-IR microscopy at a resolution better than the diffraction limit. Applied Spectroscopy 1999, 53, (7), 810-815.

12. Bozec, L.; Hammiche, A.; Pollock, H. M.; Conroy, M.; Chalmers, J. M.; Everall, N. J.; Turin, L. Localized photothermal infrared spectroscopy using a proximal probe. Journal of Applied Physics 2001, 90, (10), 5159-5165.

13. Hammiche, A.; Bozec, L.; German, M. J.; Chalmers, J. M.; Everall, N. J.; Poulter, G.; Reading, M.; Grandy, D. B.; Martin, F. L.; Pollock, M. Mid-infrared microspectroscopy of difficult samples using near-field photothermal microspectroscopy. Spectroscopy 2004, 19, (2), 20-42.

14. Hammiche, A.; Bozec, L.; Pollock, H. M.; German, M.; Reading, M. Progress in near-field photothermal infra-red microspectroscopy. Journal of Microscopy 2004, 213, (2), 129-134.

15. Dai, X.; Reading, M.; Craig, D. Q. M. Mapping amorphous material on a partially crystalline surface: Nanothermal analysis for simultaneous characterisation and imaging of lactose compacts. Journal of Pharmaceutical Sciences 2009, 98, (4), 1499-1510.

16. Harding, L.; King, W.; Dai, X.; Craig, D. M.; Reading, M. Nanoscale characterisation and imaging of partially amorphous materials using local thermomechanical analysis and heated tip AFM. Pharmaceutical Research 2007, 24, (11), 2048-2054. 
17. Zhang, J.; Bunker, M.; Chen, X.; Parker, A. P.; Patel, N.; Roberts, C. J. Nanoscale thermal analysis of pharmaceutical solid dispersions. International Journal of Pharmaceutics 2009, 380, (1-2), 170-173.

18. Dai, X.; Moffat, J. G.; Mayes, A. G.; Reading, M.; Craig, D. Q. M.; Belton, P. S.; Grandy, D. B. Thermal probe based analytical microscopy: Thermal analysis and photothermal Fourier-transform infrared microspectroscopy together with thermally assisted nanosampling coupled with capillary electrophoresis. Analytical Chemistry 2009, 81, (16), 6612-6619.

19. Moffat, J. G.; Eddleston, M. D.; Belton, P. S.; Jones, W.; Craig, D. Q. M. Analysis of single particle photodegradation using photothermal infrared microspectroscopy. Analyst 2013, 138, (8), 2315-2322.

20. Moffat, J. G.; Mayes, A. G.; Belton, P. S.; Craig, D. Q. M.; Reading, M.

Compositional analysis of metal chelating materials using near-field photothermal Fourier transform infrared microspectroscopy. Analytical Chemistry 2010, 82, (1), 91-97.

21. Harding, L.; Qi, S.; Hill, G.; Reading, M.; Craig, D. Q. M. The development of microthermal analysis and photothermal microspectroscopy as novel approaches to drug-excipient compatibility studies. International Journal of Pharmaceutics 2008, 354, (1-2), 149-157.

22. Harding, L. J.; Reading, M.; Craig, D. Q. M. The development of thermally assisted particle manipulation and thermal nanointeraction studies as a means of investigating drug-polymer interactions. Journal of Pharmaceutical Sciences 2008, 97, (4), 1551-1563.

23. Tanojo, H.; Bouwstra, J. A.; Junginger, H. E.; Boddé, H. E. Subzero thermal analysis of human stratum corneum. Pharmaceutical Research 1994, 11, (11), 16101616.

24. Watkinson, R. M.; Herkenne, C.; Guy, R. H.; Hadgraft, J.; Oliveira, G.; Lane, M. $\mathrm{E}$. Influence of ethanol on the solubility, ionization and permeation characteristics of ibuprofen in silicone and human skin. Skin Pharmacology and Physiology 2009, 22, (1), 15-21.

25. Oliveira, G.; Hadgraft, J.; Lane, M. E. The influence of volatile solvents on transport across model membranes and human skin. International Journal of Pharmaceutics 2012, 435, (1), 38-49.

26. OECD, Guidance Document for the Conduct of Skin Absorption Studies. OECD Publishing: 2004; Vol. 28.

27. Klang, V.; Schwarz, J. C.; Hartl, A.; Valenta, C. Facilitating in vitro tape stripping: Application of infrared densitometry for quantification of porcine stratum corneum proteins. Skin Pharmacology and Physiology 2011, 24, (5), 256-268. 
28. Hahn, T.; Hansen, S.; Neumann, D.; Kostka, K. H.; Lehr, C. M.; Muys, L.; Schaefer, U. F. Infrared densitometry: A fast and non-destructive method for exact stratum corneum depth calculation for in vitro tape-stripping. Skin Pharmacology and Physiology 2010, 23, (4), 183-192.

29. Herkenne, C.; Naik, A.; Kalia, Y. N.; Hadgraft, J.; Guy, R. H. Effect of propylene glycol on ibuprofen absorption into human skin in vivo. Journal of Pharmaceutical Sciences 2008, 97, (1), 185-197.

30. ICH, Validation of Analytical Procedures: Text and Methodology Q2(R1) FDA: 2005.

31. Harding, L.; Reading, M.; Craig, D. Q. M. The development of heated tip forcedistance measurements as a novel approach to site-specific characterization of pharmaceutical materials. Journal of Pharmaceutical Sciences 2008, 97, (7), 27682779.

32. Tanojo, H.; Bouwstra, J. A.; Junginger, H. E.; Boddé, H. E. Thermal analysis studies on human skin and skin barrier modulation by fatty acids and propylene glycol. Journal of Thermal Analysis and Calorimetry 1999, 57, (1), 313-322.

33. Watkinson, R. M.; Guy, R. H.; Hadgraft, J.; Lane, M. E. Optimisation of cosolvent concentration for topical drug delivery II: Influence of propylene glycol on ibuprofen permeation. Skin Pharmacology and Physiology 2009, 22, (4), 225-230.

34. Manrique, Y. J.; Pacheco, D. P.; Martínez, F. Thermodynamics of mixing and solvation of ibuprofen and naproxen in propylene glycol + water cosolvent mixtures. Journal of Solution Chemistry 2008, 37, (2), 165-181.

35. lervolino, M.; Raghavan, S. L.; Hadgraft, J. Membrane penetration enhancement of ibuprofen using supersaturation. International Journal of Pharmaceutics 2000, 198, (2), 229-238.

36. Trottet, L.; Merly, C.; Mirza, M.; Hadgraft, J.; Davis, A. F. Effect of finite doses of propylene glycol on enhancement of in vitro percutaneous permeation of loperamide hydrochloride. International Journal of Pharmaceutics 2004, 274, (1-2), 213-219.

37. Tsai, J.-C.; Cappel, M. J.; Flynn, G. L.; Weiner, N. D.; Kreuter, J.; Ferry, J. J. Drug and vehicle deposition from topical applications: Use of in vitro mass balance technique with minoxidil solutions. Journal of Pharmaceutical Sciences 1992, 81, (8), 736-743.

38. Intarakumhaeng, R.; Li, S. K. Effects of solvent on percutaneous absorption of nonvolatile lipophilic solute. International Journal of Pharmaceutics 2014, 476, (1-2), 266-276.

39. Mahrhauser, D.-S.; Nagelreiter, C.; Gehrig, S.; Geyer, A.; Ogris, M.; Kwizda, K.; Valenta, C. Assessment of Raman spectroscopy as a fast and non-invasive method for 
total stratum corneum thickness determination of pig skin. International Journal of Pharmaceutics 2015, 495, (1), 482-484.

40. Liang, L. H.; Li, J. C.; Jiang, Q. Size-dependent melting depression and lattice contraction of Bi nanocrystals. Physica B: Condensed Matter 2003, 334, (1-2), 49-53.

41. Jiang, Q.; Zhang, Z.; Li, J. C. Melting thermodynamics of nanocrystals embedded in a matrix. Acta Materialia 2000, 48, (20), 4791-4795.

42. Jones, B. A.; Torkelson, J. M. Large melting point depression of 2-3-nm lengthscale nanocrystals formed by the self-assembly of an associative polymer: Telechelic, pyrene-labeled poly(dimethylsiloxane). Journal of Polymer Science Part B: Polymer Physics 2004, 42, (18), 3470-3475.

43. Jiang, Q.; Shi, H. X.; Zhao, M. Melting thermodynamics of organic nanocrystals. Journal of Chemical Physics 1999, 111, (5), 2176-2180.

44. Jackson, C. L.; McKenna, G. B. The melting behavior of organic materials confined in porous solids. Journal of Chemical Physics 1990, 93, (12), 9002-9011.

45. Lu, H. M.; Li, P. Y.; Cao, Z. H.; Meng, X. K. Size-, shape-, and dimensionalitydependent melting temperatures of nanocrystals. The Journal of Physical Chemistry $C$ 2009, 113, (18), 7598-7602.

46. Klang, V.; Schwarz, J. C.; Lenobel, B.; Nadj, M.; Auböck, J.; Wolzt, M.; Valenta, C. In vitro vs. in vivo tape stripping: Validation of the porcine ear model and penetration assessment of novel sucrose stearate emulsions. European Journal of Pharmaceutics and Biopharmaceutics 2012, 80, (3), 604-614. 


\section{Monitoring Drug Crystallization in Percutaneous Penetration Using Localized Nanothermal Analysis and Photothermal Microspectroscopy}

Choon Fu Goh, ${ }_{,+, t \neq}$ Jonathan G. Moffat,, Duncan Q. M. Craig, , Jonathan Hadgraft, $\uparrow$ and Majella E. Lane $\uparrow$

†Department of Pharmaceutics, UCL School of Pharmacy, 29-39 Brunswick Square, London WC1N $1 \mathrm{AX}$, United Kingdom

$\ddagger$ Discipline of Pharmaceutical Technology, School of Pharmaceutical Sciences, Universiti Sains Malaysia, 11800 Minden, Penang, Malaysia

§Asylum Research, Oxford Instruments Company, Halifax Road, HP12 3SE High Wycombe, United Kingdom

* Corresponding author:

Choon Fu Goh

Discipline of Pharmaceutical Technology, School of Pharmaceutical Sciences, Universiti Sains Malaysia, 11800 Minden, Penang, Malaysia

Phone: +604-6532074. Fax: +604-6570017. Email: gohchoonfu@hotmail.com / choonfugoh@usm.my 
Figure S1 and S2 show the proposed mechanisms of the creation of the green domain and double transitions in TTM measurements.

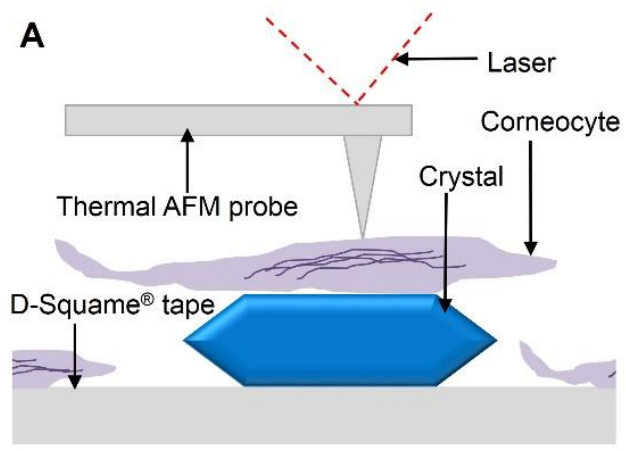

The thermal probe approaches the surface of tape until it is in contact with the corneocyte.

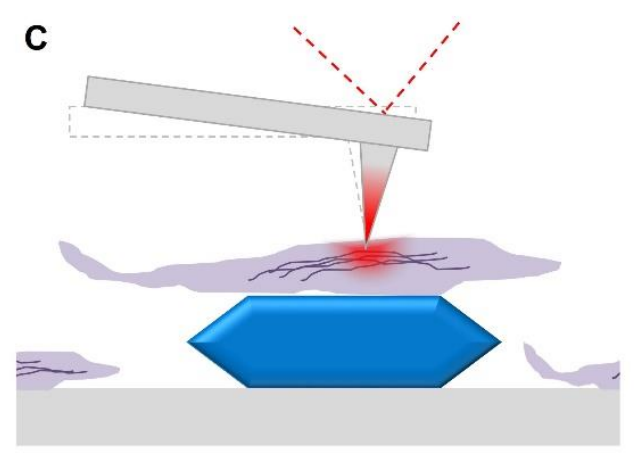

The probe deflects once the surface softens.

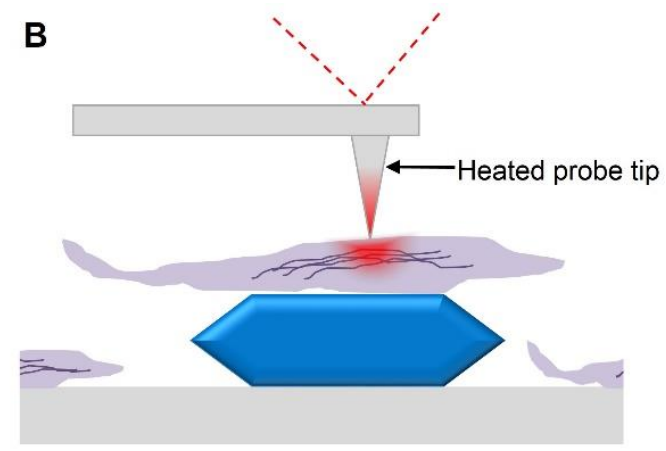

The thermal probe starts to heat up the surface of corneocyte.

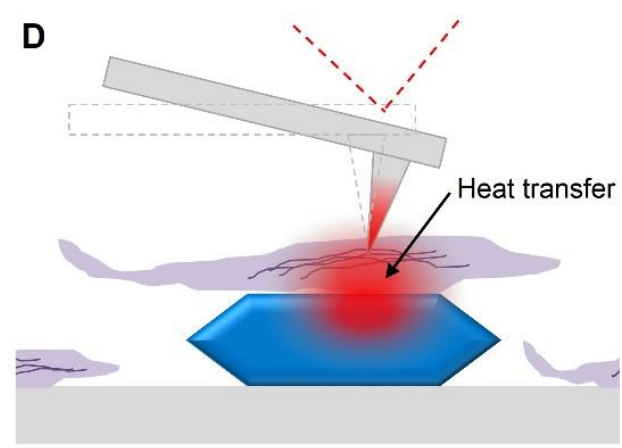

Heat transfers from the probe to the crystal through the corneocyte and softens the crystal, followed by penetration of the probe marking as a thermal event.

Figure S1 The proposed mechanism of the creation of the green domain in the TTM image scanning over a tape with PSC and crystals 
A

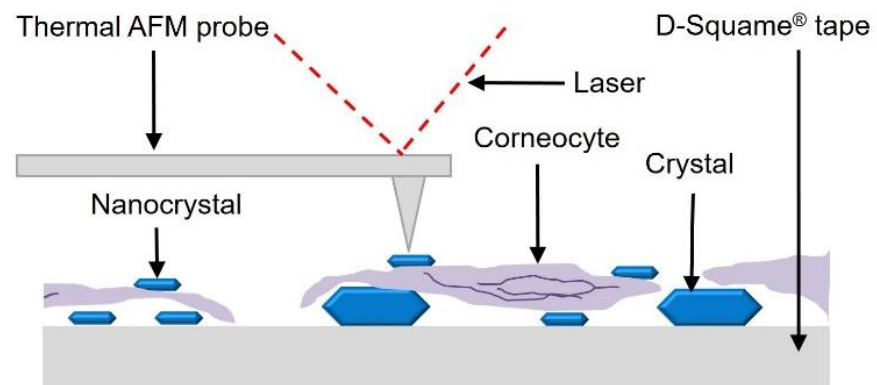

The thermal probe engages with the surface of the tape and is in contact with the nanocrystals on the corneocytes.

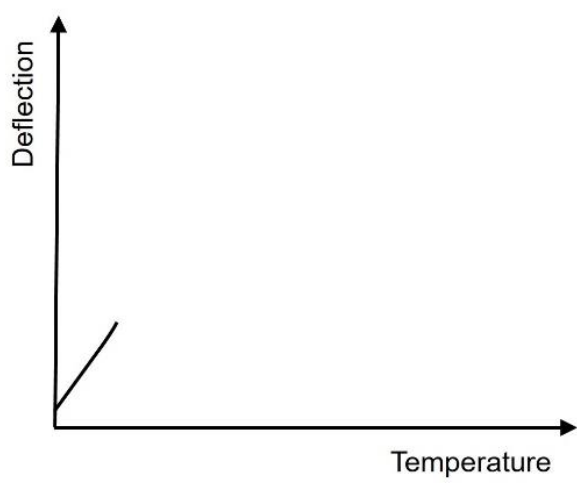

B
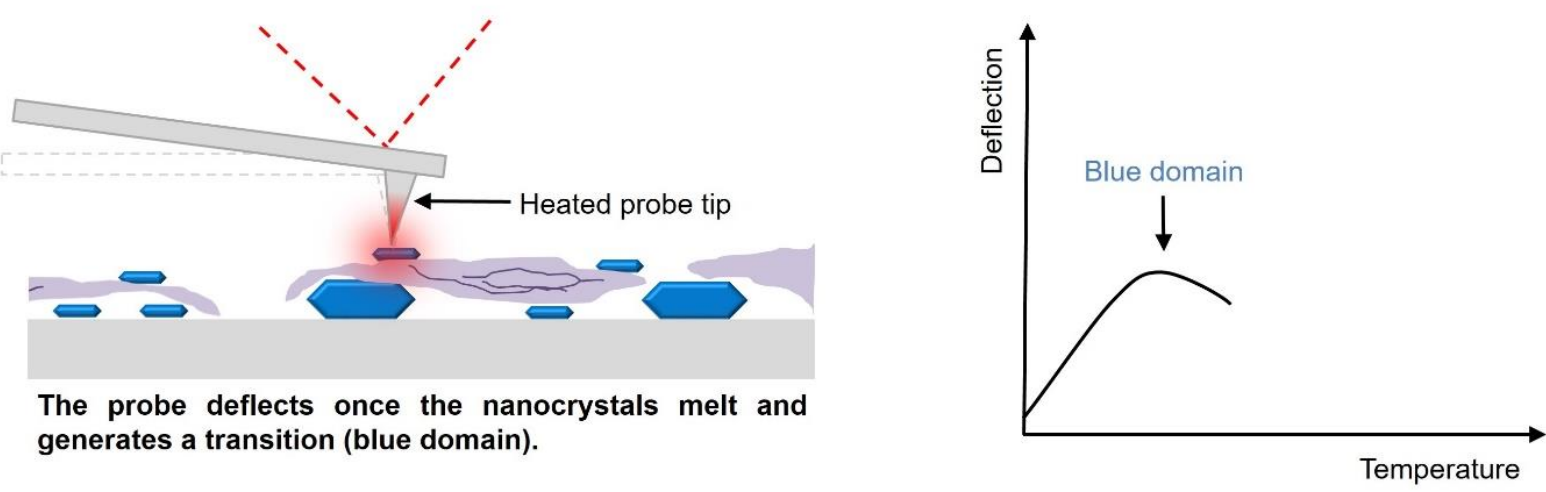

C

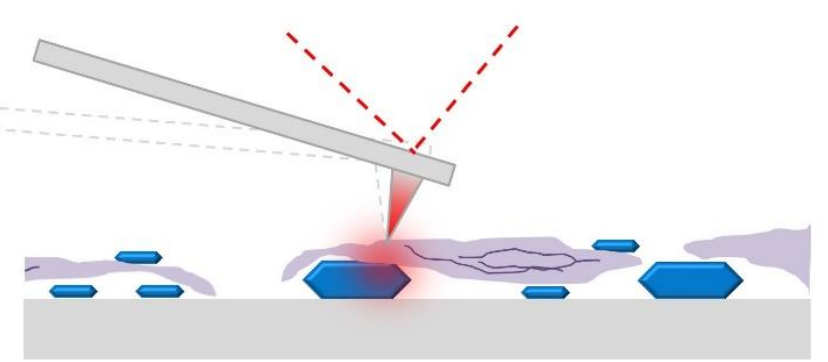

Before reaching the retraction limit, the probe continues to deflect and heats the corneocytes beneath the melted nanocrystals, resulting in the formation of a second

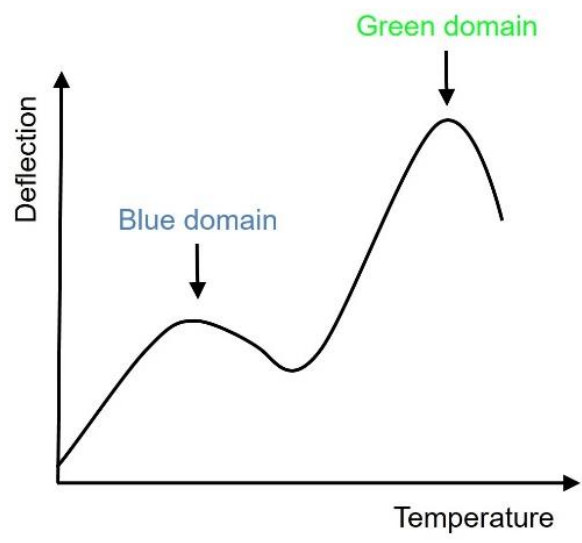
transition (green domain).

Figure S2 Schematic diagrams of the formation of double transitions in the TTM image 
Figure S3 and S4 show the PTMS measurements for untreated PSC, PSC treated with PG and IBU crystals.

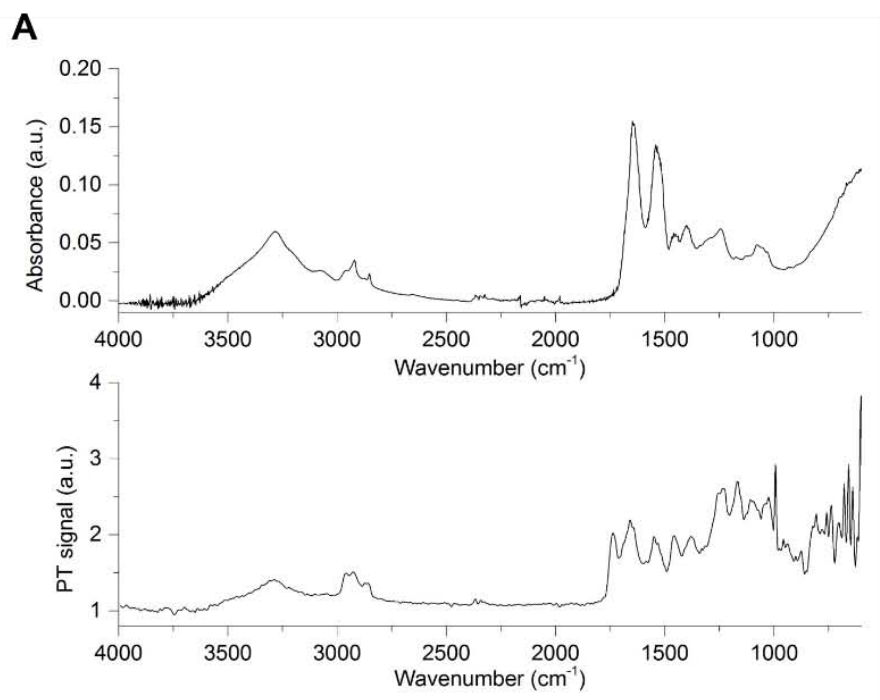

B
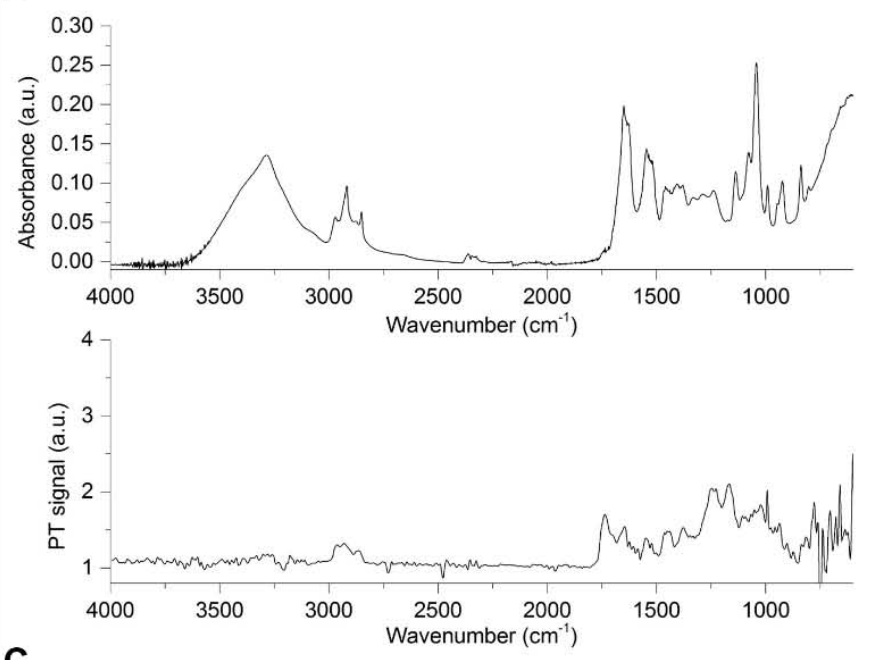

C
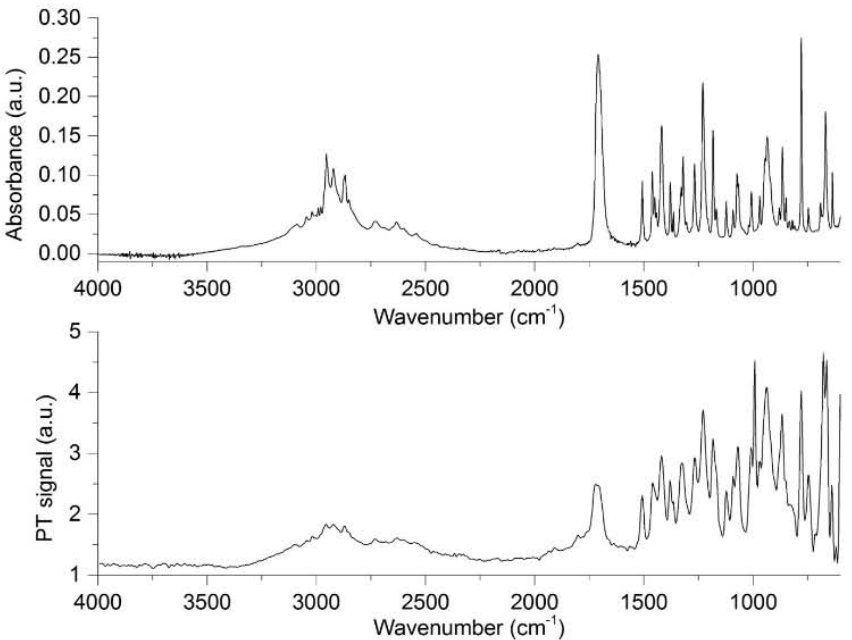

Figure S3 ATR-FTIR spectra (upper panel) and photothermal FTIR (lower panel) spectra for (A) untreated PSC, (B) PSC treated with PG and (C) IBU crystals 

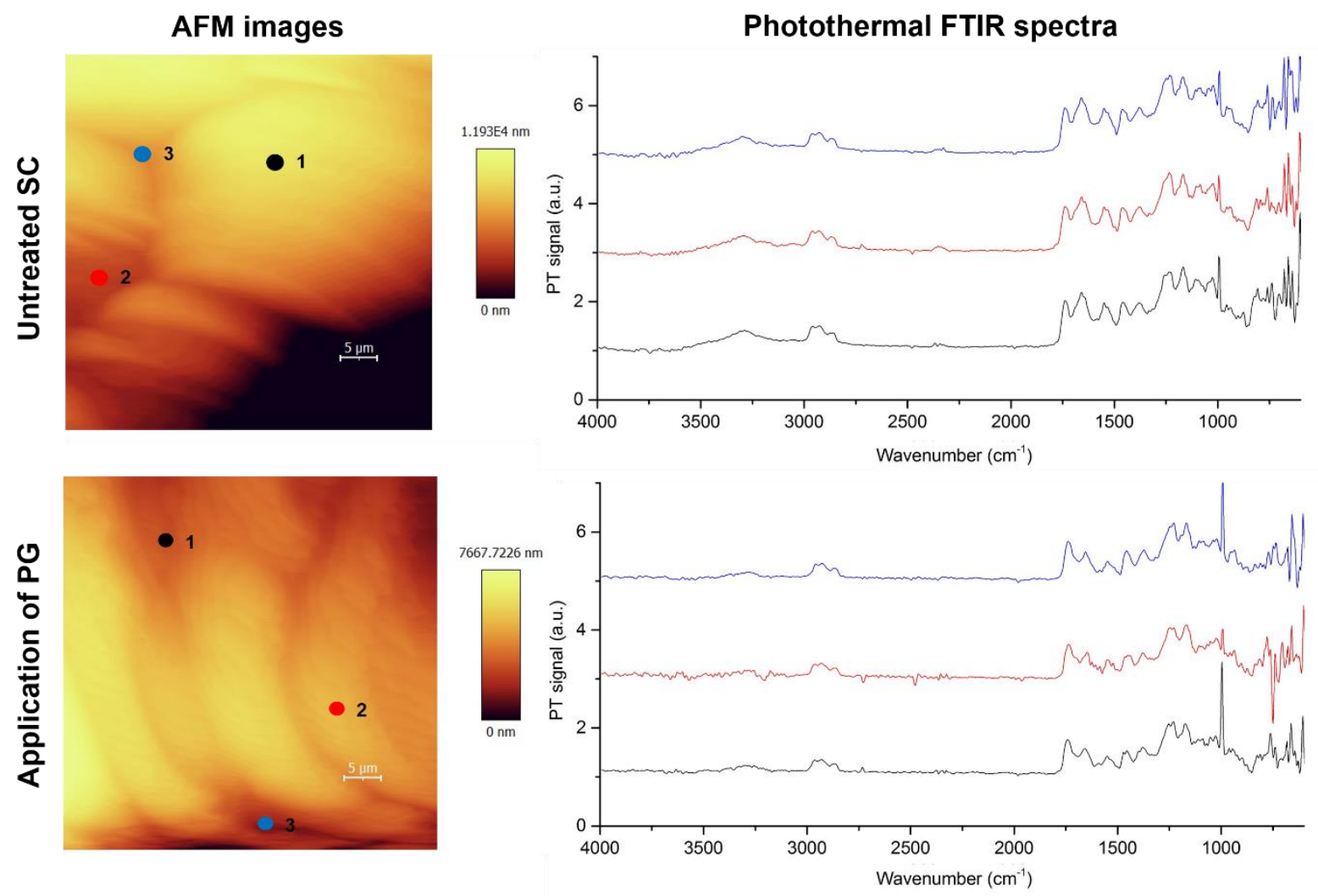

Figure S4 AFM images and corresponding photothermal FTIR spectra of PSC (with and without application of PG) on the first tape strip 